\title{
Prediction of Pre-Eclampsia by Urinary Calcium and Creatinine Ratio
}

\section{IJCRR \\ Section: Healthcare \\ Sci. Journal Impact \\ Factor: $6.1(2018)$ \\ ICV: 90.90 (2018) \\ (c) (i) (s) \\ Copyright@IJCRR}

\section{Smriti Khandelwal' ${ }^{1}$, Surekha Tayade ${ }^{2}$, Chetan Gode ${ }^{3}$}

Junior Resident, Obstetrics and Gynecology, Jawaharlal Nehru Medical College, Datta Meghe Institute of Medical Sciences (Deemed University), Sawangi, Maharashtra, India.; ${ }^{2}$ rofessor, Obstetrics and Gynecology, ]awaharlal Nehru Medical College, Datta Meghe Institute of Medical Sciences (Deemed University), Sawangi, Maharashtra, India; 3Professor, Electronics \& Tele. Engineering, Yeshwantrao Chavan College of Engineering, Nagpur, Maharashtra, India.

\section{ABSTRACT}

Background: Prediction of preeclampsia and instituting preventive measures in high-risk individuals will favourably affect pregnancy outcomes. Urinary calcium/creatinine ratio is a low-cost biochemical test for prediction of preeclampsia which has not been studied extensively especially in a rural setup. This study aims to evaluate its efficacy.

Objectives: To study urinary calcium/creatinine ratio in pregnant women of rural setup and determine its association with preeclampsia to assess it as a screening stool for its prediction.

Methods: This is a prospective observational study wherein 500 pregnant women will be recruited after informed consent before 20 weeks of gestation and urinary calcium creatinine ratio will be calculated from a spot sample. The study participants will then be followed up until their delivery to note the development of preeclampsia. A low ratio will be considered as a good predictor.

Results: The researcher will note hypertensive disorders in study subjects and classify them according to severity. Mean urinary calcium creatinine ratio will be determined and the role of its spot value for predicting preeclampsia will be evaluated. A low value is expected in women with pre-eclampsia as compared to normotensive women.

Conclusion: Depending on the study outcome, we would like to comment on the frequency of preeclampsia and the effectiveness of urinary calcium creatinine ratio in predicting it in a low resource setting.

Key Words: Pre-eclampsia, Urinary calcium, Urin creatinine, Ratio

\section{INTRODUCTION}

Preeclampsia is a multi-system condition emerging in the latter half of pregnancy or postpartum by the development of new-onset hypertension and/or proteinuria and end-organ failure when severe. The genesis of the disease is attributed to early pregnancy wherein the anatomy of spiral arterioles of the uterus at the placental site is remodelled and distorted. ${ }^{1}$ Early onset of preeclampsia causes substantial mortality and maternal and perinatal morbidity. Therefore, due to the lack of validated prophylaxis for pre-eclampsia, risk assessment or subclinical disease detection is desirable to classify patients for more intensive observation. ${ }^{2}$ Effective aspirin treatment earlier decreases the risk of pre-eclampsia, and accurate pre-eclampsia screening tests are a clinical priority. Clinical assessment of the likelihood of pre-eclampsia is generally focused mainly on maternal experience, which has a limited predictive capacity and is not relevant to nulliparous females. Numerous primary research evaluated the predictive ability of various tests. ${ }^{3}$ Yet none of them have been proven to be optimal either due to the high incidence of false positivity or their difficulty in the interpretation of tests.

Pregnancy constitutes reasons for extraordinary calcium needs and requirements. Maternal loss of calcium during pregnancy and lactation is a common part of the growth of neonates and foetuses. There is physiological compensation in the pregnant female with increased absorption of by intestines, reduction in renal loss and some reabsorption from the bones. Renal functions may be affected by symptom-free women who ultimately experience preeclampsia. ${ }^{4}$ Gestational hypocalciuria is known to increase the risk of Preeclampsia. In various types of hypertension, the literature suggests the function of irregular calcium metabolism. Epidemiological studies have shown that the occurrence of eclampsia is

\section{Corresponding Author:}

Dr. Smriti Khandelwal, F-28 Radhikabai hostel, JNMC, DMIMS, DU, Sawangi(Meghe), Wardha, Maharashtra, India. Contact: 9765467718; Email: khandelwalsmriti24@gmail.com

ISSN: 2231-2196 (Print)

Received: 28.08 .2020
ISSN: 0975-5241 (Online)

Revised: 26.09 .2020
Accepted: 04.11 .2020

Published: 30.11 .2020 
inversely proportional to the intake of calcium in the diet. Many studies have reported a decline in urinary calcium excretion in preeclampsia. Studies of high-risk population detection by urinary calcium level or calcium creatinine ratio have had conflicting results.

Ozcan et al (1995) were the first to research the predictive value of decreasing calcium and creatinine ratio in a spot urine sample as an efficient marker for pre-eclampsia. ${ }^{5}$ Another research showed that reduced urinary excretion of calcium may be considered a valuable tool for early pre-eclampsia diagnosis. ${ }^{6}$ However, no such study has been carried out in low resource and rural settings and it is our objective to do so.

Preeclampsia largely contributes to the morbidity and mortality of pregnant females and poor fetal outcome. Predicting preeclampsia can help in improving maternal and foetal health. Various biochemical tests have been studied for its prediction, however, most are complex and costly. Preeclampsia is associated with abnormal calcium ratio and urinary calcium/creatinine ratio which can be done easily at most of the centres can help in increasing the proportion of preeclampsia which can be predicted. ${ }^{7,8}$

\section{OBJECTIVES}

1. To study the urinary calcium/creatinine ratio in pregnant women.

2. To correlate the ratio with the development of preeclampsia.

3. To evaluate predictive values of urinary calcium/creatinine ratio

4. To assess the use of urinary calcium/creatinine ratio as a screening stool for predicting preeclampsia.

\section{MATERIALS AND METHODS}

Research question: Is spot urine calcium/creatinine ratio efficient as a predictive tool for preeclampsia (Table 1)

\section{Table 1: Pico Format}

\begin{tabular}{ll}
\hline $\begin{array}{l}\text { Patient population } \\
\text { and problem }\end{array}$ & $\begin{array}{l}\text { Normotensive pregnant women below } \\
\text { 2o weeks of gestation. }\end{array}$ \\
Intervention & $\begin{array}{l}\text { This is an observational study } \\
\text { wherein urinary calcium creatinine } \\
\text { ratio will be measured }\end{array}$ \\
& $\begin{array}{l}\text { Urine calcium/creatinine ratio in } \\
\text { normotensive and pre-eclamptic will } \\
\text { be compared }\end{array}$ \\
Outcome & $\begin{array}{l}\text { It is expected that urinary calcium } \\
\text { creatinine ratio will be lower in pre- } \\
\text { eclamptic }\end{array}$ \\
\hline
\end{tabular}

Ethical approval: Ethical approval will be obtained from the Institutional Ethics Committee (IEC) and written informed consent from all enrolled women.

Study design: Prospective Hospital Based Observational Study.

Duration of Study: 2 years

Study Site: Obstetrics and Gynaecology unit of AVBRH, Sawangi (Meghe), Wardha which is a rural-based tertiary institute.

Study Population: Pregnant women seeking care at an outpatient unit of Obstetrics \& Gynaecology, in the AVBRH hospital, Sawangi, Meghe, in the first trimester, fitting into inclusion and exclusion criteria and were amenable for follow up.

Sample size: 500

\section{Inclusion criteria:}

Asymptomatic pregnant women before 20 weeks of gestation having blood pressure less than $140 / 90 \mathrm{mmHg}$ and no proteinuria.

\section{Exclusion criteria:}

1. Pregnant women (PW) with a history of preeclampsia/ eclampsia, diabetes mellitus, chronic hypertension, renal diseases,

2. $\mathrm{PW}$ with twin gestation.

3. PW receiving anti-epileptic medication

Women fulfilling inclusion criteria would be recruited after ethical approval and informed written consent. Demographic characteristics, obstetric history and medical history will be noted and blood pressure will be measured in the right arm at sitting position. Exclusion of proteinuria will be done by Dip-stick process in a spot urine sample. Spot urine samples will be centrifuged and analyzed in the Institute's Biochemistry Laboratory by commercially available standardized enzymatic kits, using creatininase method urine Creatinine will be estimated and calcium/creatinine ratio will be calculated. All study subjects will be followed up during the prenatal period until delivery without intervention from the study. Women who develop hypertension with BP surpassing 140/90 mmHg on two occasions 6 hours apart with proteinuria will be labelled as Preeclampsia.

\section{EXPECTED OUTCOMES}

It is expected that women developing preeclampsia later on in pregnancy will have a low ratio of calcium and urinary creatinine as compared to women who remain normotensive during pregnancy. Sensitivity, specificity, positive and negative predictive value and efficacy will be calculated. 


\section{DISCUSSION}

Preeclampsia is an idiopathic gestational condition increasing materno-foetal morbidity and mortality and predominantly occurs as a consequence of distorted placental anatomy. Early identification of preeclampsia is a crucial challenge in obstetric practice since early prediction and recognition can help to undertake various necessary measures which can decrease the complications associated with it for the better maternal and foetal outcome. Preeclampsia and eclampsia are said to lead to a woman's death every three minutes worldwide. Many of these problems are preventable. In India, the rate of preeclampsia in pregnancies is $8-10 \%$. Various screening tools to predict preeclampsia have been researched and tried for adaptation of prophylactic measures but no single screening tool can predict preeclampsia effectively.

Previous methods of screening pregnant women based on individual risk factors for preeclampsia falsely put the majority of pregnant women into high-risk categories. Screening of only pregnant women with risk factors can also miss on pregnant women who are asymptomatic and have no risk factors. Hence a screening tool which can be employed for all pregnant women irrespective of risk factors and can predict preeclampsia which can develop later on pregnancy is efficient. Combination of various screening tools like maternal history, Doppler studies, invasive tests have been employed but its feasibility remains a question in a rural setup. In a rural setup, a non-invasive test with the good predictive value that can categorise pregnant women depending on their risk of development of preeclampsia and women can be advised on ANC follow depending on their risk status is more effective.

A screening tool for preeclampsia will have a great impact on clinical outcome. Early prediction of preeclampsia will allow timely intervention like antihypertensives, steroids, magnesium sulphate and prevention of development of eclampsia. ${ }^{7}$ Several studies were conducted which showed a positive correlation in a decrease in urinary calcium in pregnant women who developed preeclampsia later on in pregnancy when followed up till delivery as compared to pregnant women who remained normotensive till delivery. Spot urine sample to evaluate calcium and creatinine ratio (CCR) is less cumbersome to patients than $24 \mathrm{hrs}$ urine sample as it can be done on OPD basis, no loss of urine sample collection is expected as might occur with $24 \mathrm{hrs}$ urine sample collection. The decrease in CCR can serve as a risk factor for the development of preeclampsia and can be a feasible, cheap affordable biochemical investigation in this very important medical disorder affecting PW. Early interventions as a preventing measure can contribute to averting severe acute maternal illness and death

This study is expected to show a reduced urinary calcium / creatinine ratio in patients who will have preeclampsia later on in pregnancy. Thus, early prediction of pre-eclampsia can be done and measures can be taken to prevent adverse morbidities and mortality. Few study related articles were reviewed..$^{8-10}$ Mishra and Inamdar reported on deep venous thrombosis in pregnancy. ${ }^{11}$ Nakade et al studied the Status of Vitamins and Minerals in Pregnancy. ${ }^{12}$ A relationship between uric acid and creatinine in pre-diabetic and diabetic patient is reported and studies from the Global Burden of the disease are available. ${ }^{13-15}$.

\section{CONCLUSION}

Depending on the study outcome, we would like to comment on the frequency of preeclampsia and the effectiveness of urinary calcium creatinine ratio in predicting it in a low resource setting.

\section{REFERENCES}

1. Makmur S, Rachmadsyah J. Risk Factor and Biomarker of Preeclampsia. In Prediction of Maternal and Fetal Syndrome of Preeclampsia, edited by Nidhi Sharma. IntechOpen, 2019;4(3): 23-9.

2. Myatt L, Miodovnik M. Prediction of preeclampsia. In Seminars in perinatology 1999; 23(1):45-57. WB Saunders.

3. Townsend R, Khalil A, Premakumar Y, Allotey J, Snell KI, Chan $\mathrm{C}$, et al. Prediction of pre-eclampsia: Review of reviews. Ultrasound Obstet Gynecol 2019;54(1):16-27.

4. Mittal S. Calcium/Creatinine Ratio in Spot Urine Sample for Early Detection of Pre-Eclampsia. J Evol Med Dental Sci 2014; 3(4):6.

5. Kaleli B, Ozeren M, Turan C, Zorlu G. Urinary calcium to creatinine ratio for predicting preeclampsia. Am J Perinatol 1995;12(05):349-51.

6. Vahdat M, Kashanian M, Sariri E, Mehdinia M. Evaluation of the value of calcium to creatinine ratio for predicting of preeclampsia. J Mater Fet Neonat Med 2012;25(12):2793-4.

7. Belizán J, Villar J, Repke J. The relationship between calcium intake and pregnancy-induced hypertension: up-to-date evidence. Am J Obstet Gynaecol 1988;158(4):898-902.

8. Yadav S, Agrawal M, Hariharan C, Dewani D, Vadera K, Krishna N. A comparative study of the serum lipid profile of women with preeclampsia and normotensive pregnancy. J Datta Meghe Inst Med Sci Uni 2018;13(2):83.

9. Chandak LG, Lohe VK, Bhowate RR, Gandhi KP, Vyas NV. Correlation of mandibular radio morphometric indices with serum calcium and serum estradiol in pre-and post-menopausal women. Contemp Clin Dentist 2017;8(1):53.

10. Chandak LG, Lohe VK, Bhowate RR, Gandhi KP, Vyas NV. Correlation of Periodontitis with Mandibular Radiomorphometric Indices, Serum Calcium and Serum Estradiol in Postmenopausal Women: A Case-Control Study. Ind J Dental Res 2017; 28(4): 388.

11. Inamdar S, Mishra A. Deep Venous Thrombosis in Pregnancy. J South Asian Fed Obstet Gynaecol 2020;12(1): 48-50.

12. Nakade M, Jungari ML, Ambad R, Dhingra G. Status of Vitamins and Minerals in Pregnancy: Still A Point of Concern in Central India. Int J Curr Res Rev 2020;45-49. 
13. Ranjit S. Ambad, Jha RK, Butola LK, Bankar N, Singh BR, Dhok A. Relationship between Uric Acid and Creatinine in PreDiabetic and Diabetic Patients: Vidarbha Region of Maharashtra. Int J Res Pharm Sci 2020;11(3): 3412-17.

14. James SL, Castle CD, Dingels ZV, Fox JT, Hamilton EB, Liu Z, et al. Estimating global injuries morbidity and mortality: meth- ods and data used in the Global Burden of Disease 2017 study. Inj Prev 2020;26(1):125-53.

15. Murray CJL, Abbafati C, Abbas KM, Abbasi M, Abbasi-Kangevari M, Abd-Allah F, et al. Five insights from the Global Burden of Disease Study 2019. Lancet 2020;396(10258):1135-59. 